\title{
Atmospheric Properties from the 2006 Niamey Deployment and Climate Simulation with a Geodesic Grid Coupled Climate Model
}

First Quarter 2008

ARM and Climate Change Prediction Program Report

J. Mather/Pacific Northwest National Laboratory

D. Randall/Colorado State University

December 2007

Work supported by the U.S. Department of Energy,

Office of Science, Office of Biological and Environmental Research 


\section{DISCLAIMER}

This report was prepared as an account of work sponsored by the U.S. Government. Neither the United States nor any agency thereof, nor any of their employees, makes any warranty, express or implied, or assumes any legal liability or responsibility for the accuracy, completeness, or usefulness of any information, apparatus, product, or process disclosed, or represents that its use would not infringe privately owned rights. Reference herein to any specific commercial product, process, or service by trade name, trademark, manufacturer, or otherwise, does not necessarily constitute or imply its endorsement, recommendation, or favoring by the U.S. Government or any agency thereof. The views and opinions of authors expressed herein do not necessarily state or reflect those of the U.S. Government or any agency thereof. 


\section{Contents}

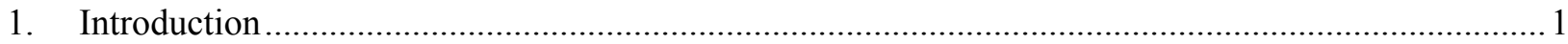

2. Atmospheric Properties from the 2006 Niamey Deployment ....................................................... 1

2.1 Cloud Occurrence Profiles from the 95-GHz Cloud Radar.................................................... 1

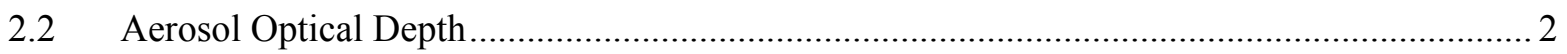

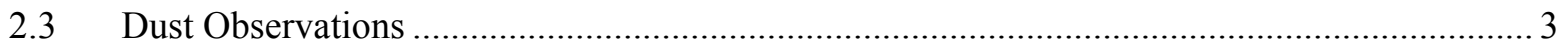

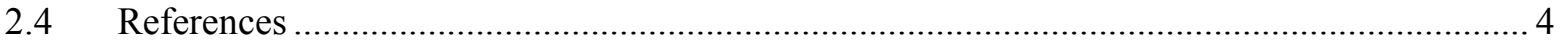

3. Initial Testing in Preparation for a Year-Long Control Simulation Using Geodesic Grid Coupled Climate Model at a Resolution $250 \mathrm{~km}$............................................................................... 4

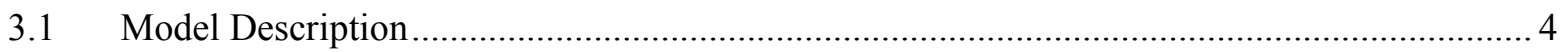

3.2 Tests of the Atmosphere and Land-Surface Models With Prescribed Sea-Surface

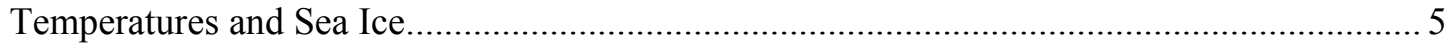

3.3 Tests of the Ocean and Sea Ice Models With Prescribed Atmospheric Forcing ...................... 6

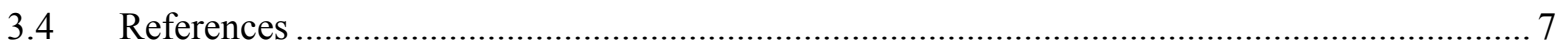

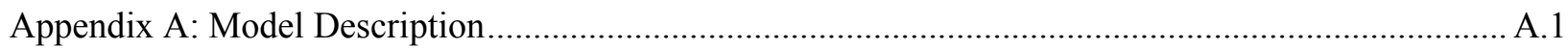

\section{Figures}

1: The process used to create a geodesic grid, by starting from an icosahedron. ................................. 4

2: The observed and simulated annual-mean distributions of total precipitation. .................................. 5

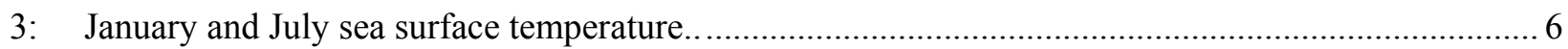




\section{Introduction}

In 2008, the Atmospheric Radiation Measurement (ARM) program and the Climate Change Prediction Program (CCPP) have been asked to produce joint science metrics. For CCPP, the metrics will deal with a decade-long control simulation using geodesic grid-coupled climate model. For ARM, the metrics will deal with observations associated with the 2006 deployment of the ARM Mobile Facility (AMF) to Niamey, Niger. Specifically, ARM has been asked to deliver data products for Niamey that describe cloud, aerosol, and dust properties. The first quarter milestone is 'initial formulation of the algorithm to produce and make available, new continuous time series of retrieved cloud, aerosol and dust properties, based on results from the ARM Mobile Facility deployment in Niger, Africa.' The first quarter milestone has been achieved and progress is reported below.

\section{Atmospheric Properties from the 2006 Niamey Deployment}

\subsection{Cloud Occurrence Profiles from the 95-GHz Cloud Radar}

Observations form the $95 \mathrm{GHz}$ W-band ARM Cloud Radar (WACR), Micropulse Lidar (MPL), and ceilometer will be combined using the new WACR Active Remote Sensing of Clouds (WACR-ARSCL) value added product (Kollias and Miller, 2007) to produce cloud boundaries and time-height profiles of cloud location, radar moments, and linear depolarization ratio (LDR) fields. The temporal resolution will be 5 seconds. The vertical resolution is equal to that of the WACR, $42.856 \mathrm{~m}$.

The basic algorithm used in the WACR-ARSCL value added product (VAP) is similar to that employed by the ARSCL VAP, which is based on 35-GHz Millimeter Cloud Radar observations (Clothiaux et al., 2000). First, an MPL-based cloud mask is determined based on the comparison of lidar backscatter measurements to returns during known clear-sky periods. Next, a WACR cloud and precipitation mask is derived from signal-to-noise ratio thresholds determined for each time profile. The MPL cloud mask is combined with ceilometer cloud base estimates to produce a best-estimate cloud base for each time. The MPL and WACR cloud masks are merged, then additional filtering of the resulting cloud mask is done in the lower troposphere (below approximately $3.5 \mathrm{~km}$ ) to remove insect returns. Insects are identified using a combination of WACR LDR and reflectivity measurements.

\subsubsection{Data Streams}

The WACR-ARSCL VAP will produce two data streams, each having one file per day. The first data stream will contain time sequences of cloud boundaries, including ceilometer cloud base, MPL/ceilometer best-estimate cloud base, radar-derived first cloud top, and combined radar-MPL cloud base and top for up to 10 cloud layers for each time. The second data stream will contains the cloud boundary information in the above data stream, plus a number of time-height fields, including the merged radar-MPL cloud mask, original and masked WACR reflectivity, and masked mean Doppler velocity, spectral width, and LDR. The cloud mask field indicates the instrument source(s) for each positive cloud detection. For each WACR-ARSCL data stream, the cloud mask indicates whether or not a cloud has been detected at a given altitude and time. 
From these daily files, a single annual file will produced that reports the cloud frequency over the Niamey site as a function of time and altitude. The cloud frequency will be calculated as the fraction of time a cloud is observed in the current time interval at a particular altitude.

\subsection{Aerosol Optical Depth}

The Multi-Filter Rotating Shadowband Radiometer (MFRSR) Optical Depth VAP uses a technique developed by Joseph Michalsky, et al (2001) as described in the Journal of Geophysical Research, June 2001. This method uses Langley regression data as computed by the Langley VAP (documented elsewhere) to obtain daily top of atmosphere solar radiance values (referred to as $I_{0}$ ) representative of the hypothetical response of the MFRSR in the absence of atmospheric effects. These daily $\mathrm{I}_{\mathrm{o}}$ values are then used to compute optical depths from MFRSR data at 15-second temporal resolution. Optical depths are computed at five wavelengths for each non-cloudy MFRSR measurement.

\subsubsection{The Algorithm and Methodology}

The method described by Michalsky et al has been modified to yield optical depth values for each noncloudy time record, rather than a single optical depth for a given day. The basic technique is to obtain many Langley Io values on either side of the day for which the optical depths will be computed. Currently this is approximately 30 days before and 30 days after the date being computed. This set of Langley data is then processed by a modified Forgan technique to remove outliers. The remaining $\mathrm{I}_{0} \mathrm{~s}$ are then smoothed using a lowess regression over the entire time range. The $I_{0}$ values for a given day are then used to compute an optical depth for each measurement time.

\subsubsection{Input Data Sources}

There are two primary data sources for this VAP.

- Langley VAP output (Langley Io data)

- MFRSR or NIMFR data (direct normal narrowband data).

If available, the following auxiliary data are also used:

1. TOMS satellite data (ozone data)

2. Some source for atmospheric pressure (ARM site/facility specific).

\subsubsection{Output Products}

The optical depth VAP produces a single netCDF file per day. This file contains the optical depths for each MFRSR or NIMFR sample, for these primary narrowband filters:

- $415 \mathrm{~nm}$ (referred to as "filter1" in the netCDF file)

- $500 \mathrm{~nm}$ (referred to as "filter2")

- $615 \mathrm{~nm}$ (referred to as "filter3")

- $673 \mathrm{~nm}$ (referred to as "filter4")

- $870 \mathrm{~nm}$ (referred to as "filter5")

- Angstrom exponent. 
In the absence of clouds, these optical depths correspond to column aerosol optical depths at each wavelength.

From the daily files, a single-file time series of optical depth for the six wavelengths will also be constructed.

\subsection{Dust Observations}

There is not an existing dust value added product currently run for any of the ARM Climate Research Facility (ACRF) sites. This is due in part because dust has not been a major factor at the other sites. However, at Niamey, significant dust events have sparked a lot of scientific interest and give motivation for identifying when these events occur. At Niamey, the two predominate types of aerosol are biomass and dust. The purpose of this new data product will be to identify when dust is dominating the aerosol optical depth so that the properties of these dust events can be further studied. In order to identify these events, we will augment the column optical depth measurements derived from the MFRSR with in situ measurements.

The aerosol observing system (AOS) is the primary Atmospheric Radiation Measurement (ARM) platform for in situ aerosol measurements at the surface. The principal measurements are optical absorption and scattering coefficients at one and ten micron particle size ranges and at three wavelengths, nominally red, green, and blue. Additional measurements include those of the particle number concentration, size distribution, hygroscopic growth, and inorganic chemical composition. The AOS measures aerosol optical properties to understand how particles interact with solar radiation and influence the earth's radiation balance. The measurements are useful for calculating parameters used in radiative forcing calculations such as the aerosol single-scattering albedo, asymmetry parameter, mass scattering efficiency, and hygroscopic growth.

The dust storms at Niamey have several characteristic signatures that will be used to identify these events. The most important of these is the relative size of the dust particles and the degree to which the large particles dominate the total aerosol scattering measurements. Almost as important is the observation that the dust particles have significant absorption (SSA $<.9$ for large particle scattering fraction) but not nearly so much as soot due to biomass burning (SSA $\sim 0.8$ ).

In order to detect dust storms aloft that have not yet impacted the ground site, these AOS measurements will be augmented with MFRSR Aerosol Optical Depth measurements having high optical depth and near-zero angstrom exponent - again an indication of extinction dominated by large particles in the column.

The main output of this dust product will be a flag indicating when dust is the dominant form of aerosol. This flag will be coupled with the optical depth to provide radiative property information about the dust when these events occur. These dust parameters will be reported as a time series in a single file. 


\subsection{References}

Clothiaux, E. E., T. P. Ackerman, G. G. Mace, K. P. Moran, R. T. Marchand. M. A. Miller, B. E. Martner, 2000: Objective Determination of Cloud Heights and Radar Reflectivities Using a Combination of Active Remote Sensors at the ARM CART Sites. J. Appl. Meteor., 39, 645-665.

Kollias, P. and M. A. Miller: Cloud and Precipitation Observations at Niamey During the 2006 ARM Mobile Facility Deployment. Submitted to Geophys. Res. Lett.

Michalsky, JJ , JA Schlemmer, WE Berkheiser, JL Berndt, LC Harrison, NS Laulainen, NR Larson, and JC Barnard, 2001: Multiyear Measurements of Aerosol Optical Depth in the Atmospheric Radiation Measurement and Quantitative Links Programs." J. Geophys. Res. 106: 12,099.

\section{Initial Testing in Preparation for a Year-Long Control Simulation Using Geodesic Grid Coupled Climate Model at a Resolution $\sim 250 \mathrm{~km}$}

The annual target is to report results of decade-long control simulation using geodesic grid coupled climate model. The first quarter milestone is 'initial testing in preparation for a year-long control simulation using geodesic grid coupled climate model at a resolution $\sim 250 \mathrm{~km}$.' The first quarter milestone has been achieved and progress is reported below.

\subsection{Model Description}

The Coupled Colorado State Model (CCoSM) is a climate model in which each component is discretized on a geodesic grid (Fig. 1). A geodesic grid consists of hexagons and pentagons. The grid-cells are relatively uniform across the globe, varying in area by only $5 \%$, and the grid is quasi-isotropic. The distinct climate components in CCoSM are the atmosphere, ocean and sea ice, and their coupling is coordinated by a coupler component which computes the interfacial fluxes and PBL physics. Further description of the model is given in the Appendix.

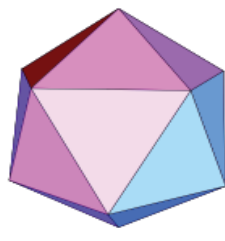

Icosahedron
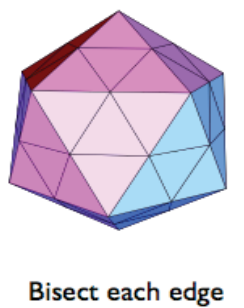
and connect the dots

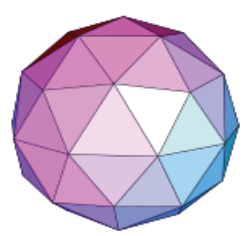

Pop out onto the unit sphere
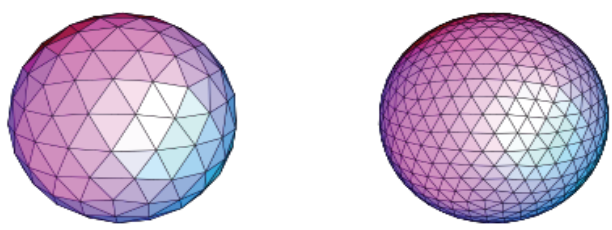

And so on, until we reach our target resolution...

Figure 1: The process used to create a geodesic grid, by starting from an icosahedron. 


\subsection{Tests of the Atmosphere and Land-Surface Models With Prescribed Sea-Surface Temperatures and Sea Ice}

The atmosphere and land-surface models have been subjected to many tests over a period of years. In a particularly important test, we have performed an "AMIP" simulation (the acronym stands for “Atmospheric Model Intercomparison Project;" Gates, 1992), in which observed sea-surface temperatures and sea-ice distributions for the years 1979-1988 were prescribed as input. The model results are then analyzed to see if the simulated atmosphere and land surface respond to the year-to-year variations in the sea-surface temperature and sea ice in the same way as observed in the real world. We used $250 \mathrm{~km}$ grid spacing.

An example of the results from our AMIP run is shown in Fig. 2, which compares simulated and observed annual-mean total precipitation.

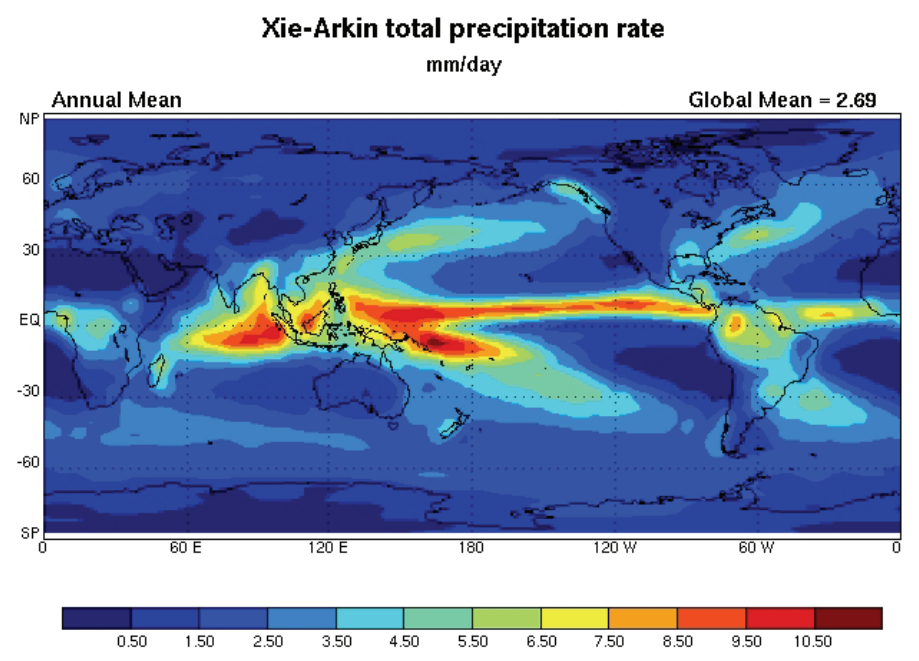

TOTAL PRECIPITATION RATE

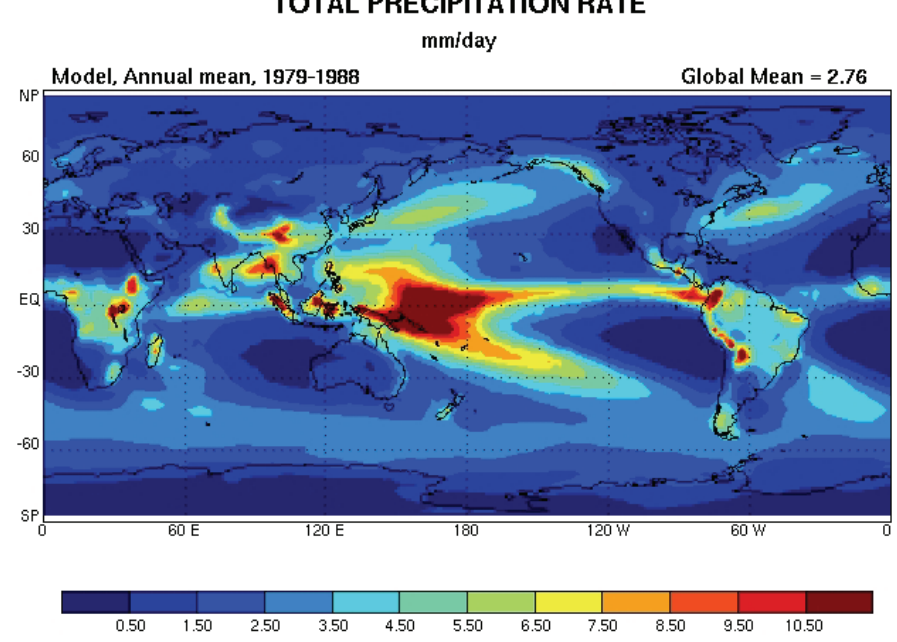

Figure 2: The observed (top panel) and simulated (bottom panel) annual-mean distributions of total precipitation. The simulation is based on an AMIP run, as described in the text. 


\subsection{Tests of the Ocean and Sea Ice Models With Prescribed Atmospheric Forcing}

To test the ocean and sea-ice models, we have performed simulations in which the ocean and sea ice are "forced" with prescribed atmospheric data.

The initial conditions for the ocean are rest (no currents), with temperature and salinity from the Levitus (1982) January climatology. The sea ice is initialized with a realistic January 1 distribution of 95\% (90\%) concentration for the northern (southern) hemisphere, with a thickness of $2 \mathrm{~m}(1 \mathrm{~m}), 0.2 \mathrm{~m}$ of snow cover, and an ice-surface temperature of $-1{ }^{\circ} \mathrm{C}$, and an interior ice snow energies consistent with temperatures of $0^{\circ} \mathrm{C}$. The atmospheric driving data is from the ERA-40 reanalysis (Uppala et al., 1999), interpolated to the geodesic grid. The model was integrated for four years with a $200 \mathrm{~s}$ time step for all components. We used $250 \mathrm{~km}$ grid-spacing.

Fig. 3 shows the mean January and July sea surface temperature maps from the NOAA data (1971 - 2000 mean), and the simulation (four year mean). The tropical patterns are well reproduced, cold in the eastern part of the basins and warmer in the west, but the cold bias in the tropics is evident.
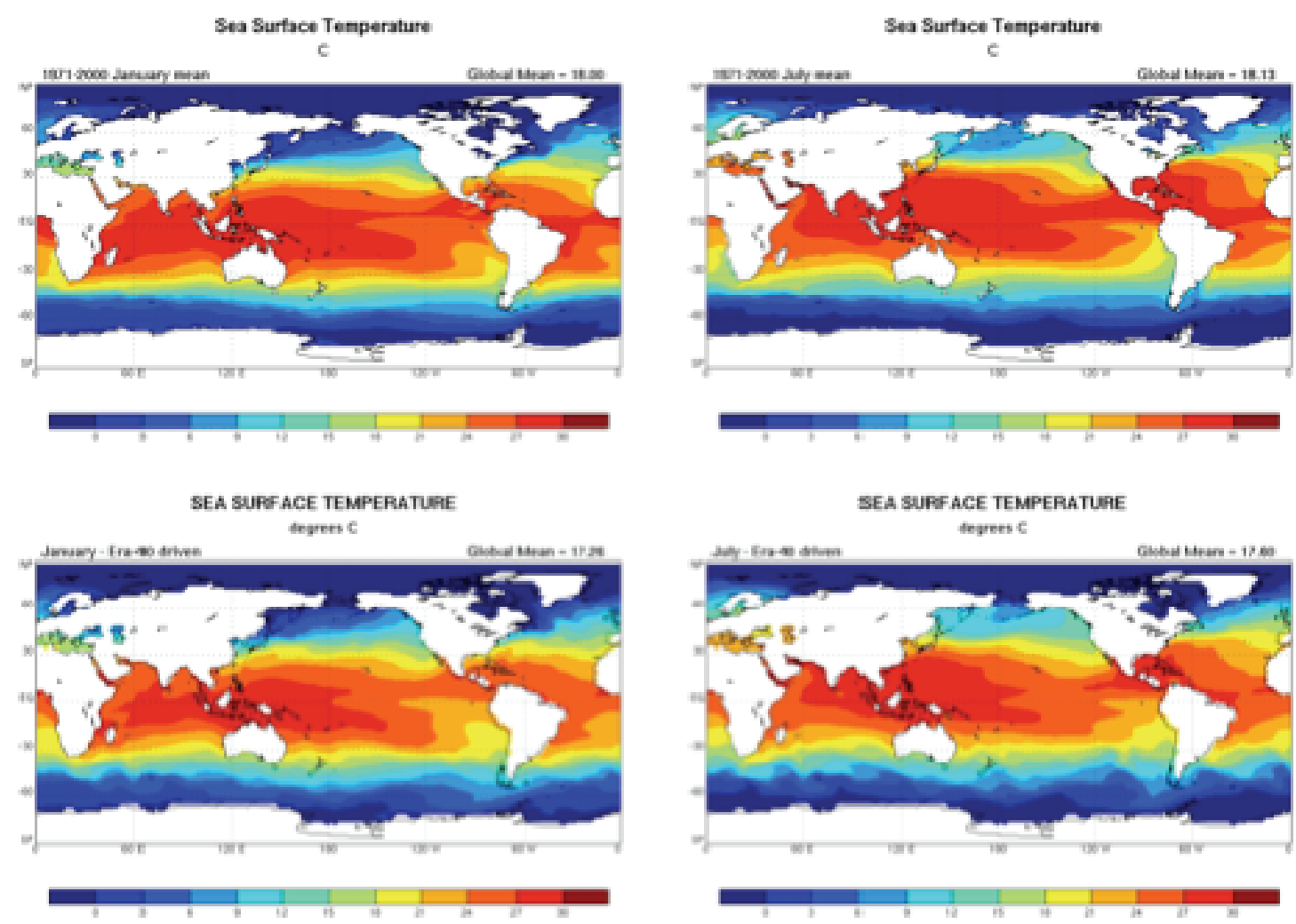

Figure 3: January (left column) and July (right column) sea surface temperature. At the top is the 1971-2000 mean from NOAA, in the middle is the 4 year mean from the data-driven ocean and sea ice models, and at the bottom the fully coupled model 2 year mean. 
The tests described above set the stage for a simulation in which all components of the model are coupled together.

\subsection{References}

Ding, P., and D. A. Randall, 1998: A Cumulus Parameterization With Multiple Cloud Base Levels. J. Geophys. Res., 103, 11341-11354.

Fowler, L. D., D. A. Randall, and S. A. Rutledge, 1996: Liquid and Ice Cloud Microphysics in the CSU General Circulation Model. Part 1: Model Description and Simulated Microphysical Processes. J. Climate, 9, 489-529.

Gates, W. L., 1992: AMIP: The Atmospheric Model Intercomparison Project. Bull. Amer. Meteor. Soc., 73, $1962-1970$.

Heikes, R. P., and D. A. Randall, 1995: Numerical Integration of the Shallow Water Equations on a Twisted Icosahedral Grid. Part I: Basic Design and Results of Tests. Mon. Wea. Rev., 123, 1862-1880.

Hunke, E. C., and J. K. Dukowicz, 1997: An Elastic-Viscous-Plastic Model for Sea Ice Dynamics. $J$. Phys. Oceanogr., 27, 1849-1867.

Jones, P. W., 1999: First- and Second-order Conservative Remapping Schemes for Grids in Spherical Coordinates. Mon. Wea. Rev., 127, 2204-2210.

Large, W. G., J. C. McWilliams, and S. C. Doney, 1994: Oceanic Vertical Mixing: a Review and a Model With a Nonlocal Boundary Layer Parameterization. Rev. Geophys., 32, 363-403.

Levitus, S., 1982: Climatological Atlas of the World Oceans. NOAA Prof. Paper 13, U. S. Govt. Printing Office, Washington, D.C.

Palmer, T. N., G. J. Shutts, and R. Swinbank, 1986: Alleviation of a Systematic Westerly Bias in General Circulation and Numerical Weather Prediction Models Through an Orographic Gravity Wave Drag Parametrization. Quart. J. R. Met. Soc., 112, 1001-1039.

Pan, D.-M., and D. A. Randall, 1998: A Cumulus Parameterization with a Prognostic Closure. Quart. J. Roy. Met. Soc., 124, 949-981.

Randall, D.A., Ringler, T.D., Heikes, R.P., Jones, P. and Baumgardner, J., 2002: Climate Modeling With Spherical Geodesic Grids, Computing in Science Engr., 4, 32-41.

Ringler, T. D., R. P. Heikes, and D. A. Randall, 2000: Modeling the Atmospheric General Circulation Using a Spherical Geodesic Grid: A New Class of Dynamical Cores. Mon. Wea. Rev., 128, 2471-2490.

Sellers, P. J., D. A. Randall, G. J. Collatz, J. Berry, C. Field, D. A. Dazlich, C. Zhang, and L. Bounoua, 1996: A Revised Land-Surface Parameterization (SiB2) for Atmospheric GCMs. Part 1: Model

Formulation. J. Climate, 9, 676-705. 
Semtner, A. J., Jr., 1976: A Model for the Thermodynamic Growth of Sea Ice in Numerical Investigations of Climate. J. Phys. Oceanogr., 6, 379-389.

Stephens, G. L., P. M. Gabriel, and P. T., Partain, 2001: Parameterization of Atmospheric Radiative Transfer. Part I: Validity of Simple Models. J. Atmos. Sci., 58, 3391 - 3409.

Uppala S., J. K. Gibson, M. Fiorino, A. Hernandez, P. Kållberg, X. Li, K. Onogi, and S. Saarinen, 1999: ECMWF Second Generation Reanalysis-ERA40. Proc. Second WCRP Int. Conf. on Reanalyses, Wokefield Park, United Kingdom, WCRP, 9-13. 


\section{Appendix A: Model Description}

The geodesic grid can be decomposed into logically rectangular subdomains, which are used to do memory allocation on a computer. Each subdomain can be decomposed in two dimensions. The model runs in a multiple-processor environment using the message passing interface (MPI).

The atmosphere sub-model includes prognostic equations for potential temperature, vorticity and divergence, surface pressure, specific humidity, cumulus kinetic energy (CKE), mixing ratios of cloud water, cloud ice, rain and snow, and the planetary boundary layer (PBL) depth. The discretization of the advection is highly conservative. The vertical coordinate is a generalized sigma coordinate, in which the PBL top is a coordinate surface. There are 29 layers, with the model top at $1 \mathrm{mb}$.

Deep moist convection is parameterized using a modified Arakawa-Schubert scheme with ice, prognostic CKE, cumulus friction, and multiple cloud bases (Pan and Randall, 1998; Ding and Randall, 1998). The large-scale cloud microphysical scheme is the one developed by Fowler et al. (2006), with cumulus detrainment as a source of cloud water and/or ice. The radiation is the Stephens parameterization. Gravity-wave drag is parameterized with a simple Palmer-like scheme (Palmer et al., 1986). Further details are given by Ringler et al. (2000) and Randall et al. (2002).

The ocean sub-model has prognostic equations for momentum, temperature, salinity and the free surface height. Depth is used as the vertical coordinate, with 33 layers. Horizontal transport is done by monotone flux-corrected transport, and vertical transport by monotone remapping. KPP (Large et al., 1994) is used to parameterize the ocean boundary-layer turbulence and convection.

The sea ice sub-model predicts ice concentration, volume and energy content. There are four ice layers, and snow is accumulated on the ice. The dynamics are based on the elastic-viscous-plastic rheology (Hunke and Dukowicz, 1997). The thermodynamics are based on Semtner (1976). Flux-corrected transport is used to advect the ice.

In addition, CCoSM includes a sub-model for land-surface processes, SiB2, which was developed by Sellers et al. (1996). SiB2 includes parameterizations of canopy physiological responses (photosynthesis, stomatal conductance), and was designed to utilize satellite measurements for many of the important vegetation boundary conditions such as fraction of short wave radiation absorbed, leaf area index, albedo and roughness.

The integrations of the these several components are coordinated by a software component called a coupler. On every time step, variables needed to compute the fluxes of mass, momentum and energy between components are passed to the coupler. The coupler computes these fluxes and sends them to the components. To deal with possible differences in resolution, a conservative interpolation is performed using SCRIP, which was developed at the Los Alamos National Laboratory (Jones, 1999). The PBL parameterization, which determines the surface fluxes, PBL-top entrainment rate, and PBL-cloudiness, is implemented in the coupler.

The coupler can also be used to replace climate components with prescribed data. For instance, the dynamic ocean and sea ice modules can be replaced with prescribed sea surface temperature and ice cover 
to drive the atmosphere; the dynamic atmosphere can be replaced with prescribed surface air conditions, radiative fluxes and precipitation to drive the ocean. In the tests described below, the coupler was used in this way 